\title{
Re-evaluation of the distribution of Meissner's corpuscles in human skin
}

\author{
Sang Hyun $\mathrm{Kim}^{1}$, Young Ho Lee ${ }^{2}$ \\ ${ }^{1}$ Department of Anatomy, Graduate School, Chungnam National University, Daejeon, ${ }^{2}$ Department of Anatomy, College of Medicine, Chungnam \\ National University, Daejeon, Korea
}

\begin{abstract}
Meissner's corpuscles are generally considered to be located in the dermal papilla of hairless skin on the fingers, toes, palms, soles, lips, eyelids, nipples, and genital organs. We used hematoxylin and eosin staining to examine the distribution of Meissner's corpuscles in skin tissues of the fingertips, palms, lips, nipples, and labia majora and minora obtained from cadavers. Many Meissner's corpuscles were observed in the dermal papilla of the fingertips, whereas the palms had only $20 \%$ as many. Meissner's corpuscles were rare in the lips, nipples, and external genital organs, which have relatively high two-point discrimination. Because Meissner's corpuscles are rapidly adapting mechanoreceptors, they may quickly detect changes in tactile sensation, including two-point discrimination, in the movable glabrous skin. In conclusion, Meissner's corpuscles might be rare in non-movable glabrous skin compared to the fingertips and palms.
\end{abstract}

Key words: Mechanoreceptors, Distribution, Skin, Two-point discrimination

Received April 29, 2020; 1st Revised May 28, 2020; 2nd Revised June 1, 2020; Accepted June 1, 2020

\section{Introduction}

Meissner' corpuscles are tactile corpuscles located in the dermal papillae of hairless skin, and are generally believed to be distributed in the fingertips, toe tips, palms, soles, lips, eyelids, nipples, and genital organs. A Meissner's corpuscle is egg-shaped, with an $80-\mu \mathrm{m}$-long axis perpendicular to the skin surface and a width of about $40 \mu \mathrm{m}$. The corpuscle consists of flattened supportive cells, Schwann cells, laid horizontally and forming lamellar discs, a distinct feature of the Meissner's corpuscle. Each corpuscle is surrounded by a connective tissue capsule. Myelinated nerve fibers enter and branch into unmyelinated nerve fibers between the cells [1,2]. The external capsule is linked to both the lamellar discs and

\section{Corresponding author:}

Young Ho Lee (iD

Department of Anatomy, College of Medicine, Chungnam National University, Daejeon 35015, Korea

E-mail:yhlee@cnu.ac.kr epidermis via collagen fibers [3].

Meissner's corpuscles are very sensitive, rapidly adapting mechanoreceptors that are mainly responsible for twopoint discrimination. Some reports suggest that they also function to detect light touch $[4,5]$, which may be associated with their distribution in sensitive areas of the lips, eyelids, nipples, and genital organs.

Meissner discovered tactile corpuscles in 1853, and researchers have subsequently studied their structure and distribution. The development of electron microscopy revealed the microscopic structure of Meissner's corpuscles. However, their distribution remains unclear [6-8].

Because many Meissner's corpuscles are on the hands and feet, most studies have examined them in the fingertips and palms. Differences in the distribution of Meissner's corpuscles have been reported between the hands of males and females, by finger region, and in patients with neurological diseases [9-11].

We frequently observed that Meissner's corpuscles were distributed in the fingertips and palms in the other study. However, few Meissner's corpuscles have been observed in

\section{Copyright () 2020. Anatomy \& Cell Biology}

This is an Open Access article distributed under the terms of the Creative Commons Attribution Non-Commercial License (http://creativecommons.org/licenses/by-nc/4.0/) which permits unrestricted non-commercial use, distribution, and reproduction in any medium, provided the original work is properly cited. 
other skin tissues such as that of the lips, eyelids, nipples, or genital organs. There are two possible explanations for these findings. First, Meissner's corpuscles may exist in all of these areas, but we have not simply observed them in sufficient tissue specimens, or they may not exist other than in the fingertips and palms. Therefore, we examined these hypotheses by observing the distribution of Meissner's corpuscles in various tissues.

This required observing as much tissue area as possible. The distribution of Meissner's corpuscles is related to the sensory functions of the body. The association between the distribution and function of Meissner's corpuscles was also investigated to re-evaluate the distribution of Meissner's corpuscles in human skin.

\section{Materials and Methods}

\section{Materials}

Tissues were obtained from the fingertips, palms, lips, nipples, and labia majora and minora of five cadavers donated for medical research and education to the Department of Anatomy, College of Medicine, Chungnam National University, Korea. All five were women, and the average age was 67.3 years (range, $53-75$ years).

\section{Methods}

Using a scalpel and scissors, tissue from the body was dissected into $1-\mathrm{cm}^{2}$ pieces including the epidermis and dermis. The samples were post-fixed for 1 day in $10 \%$ formalin and then embedded in paraffin in the vertical direction relative to the skin. Beginning where both the epidermis and dermis appeared, they were sectioned continuously to a thickness of $5 \mu \mathrm{m}$, with up to 400 sequential sections from each specimen. Hematoxylin-eosin staining was performed on the tissue slides.

The stained tissues were observed repeatedly by three re- search assistants, who checked all Meissner's corpuscles and corpuscle-like structures in the tissues. All tissue slides with Meissner's corpuscles and corpuscle-like structures were photographed (magnification, $\times 100$ ). The images were modified to $50 \%$ opacity using Adobe Illustrator and then overlaid with previous photos, counting a Meissner's corpuscle as the same one if it was in the same location.

We counted number of Meissner's corpuscles with the photograph images using Adobe Illustrator program in the consecutive sections. The observed total area was approximately $20 \mathrm{~mm}^{2}$ (5 $\mu \mathrm{m} \times 10 \mathrm{~mm} \times 400$ sheet). The density (/ $\mathrm{mm}^{2}$ ) of Meissner's corpuscles was calculated by observed total number of the corpuscles divided by 20 .

\section{Results}

Many Meissner's corpuscles were observed in the dermal papilla of the fingertips $\left(5.92 / \mathrm{mm}^{2}\right)$, with fewer in the palms $\left(1.15 / \mathrm{mm}^{2}\right.$, about $20 \%$ the number in the fingertips) (Fig. 1, Table 1). However, no Meissner's corpuscles were observed in the dermal papilla of the nipples or labia majora (Fig. 2A, $\mathrm{B}$, Table 1), and only few Meissner's corpuscle-like structures were observed in the lips and labia minora (Fig. 2C, D, Table 1).

Table 1. The number of Meissner's corpuscles in various skin tissue

\begin{tabular}{lcc}
\hline \multicolumn{1}{r}{ Region } & Sample No. & No. of Meissner's corpuscle \\
\hline Fingertips & 5 & $5.92 \pm 2.16$ \\
Palms & 5 & $1.15 \pm 0.78$ \\
Lips & 5 & $0.12 \pm 0.07$ \\
Nipples & 5 & Not observed \\
Labia majora & 5 & Not observed \\
Labia minora & 5 & $0.05 \pm 0.04$ \\
\hline
\end{tabular}

Values are presented as mean $\pm \mathrm{SD}\left(/ \mathrm{mm}^{2}\right)$.
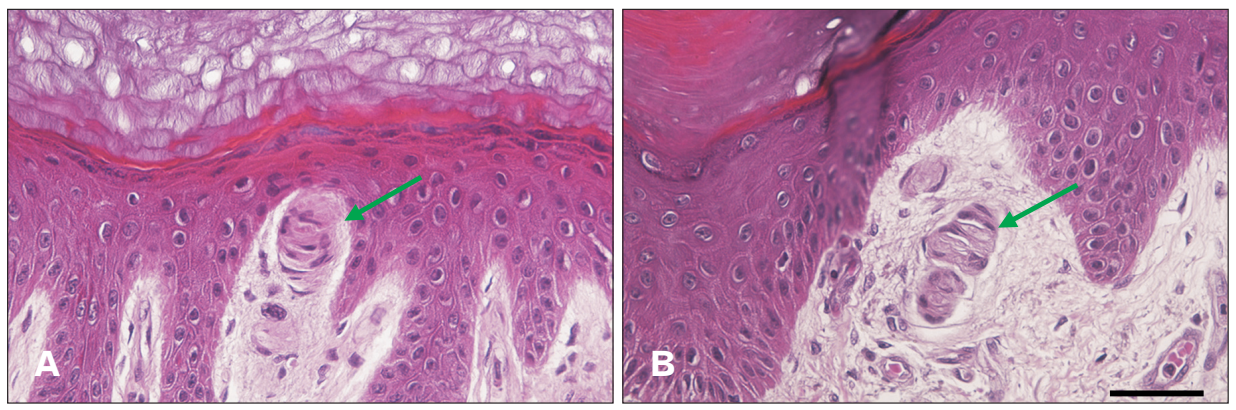

Fig. 1. Many Meissner's corpuscles were found in the dermal papillae of the fingertips (A) and palms (B) (arrow). Meissner's corpuscle, Scale bars $=50 \mu \mathrm{m}(A, B)$. 

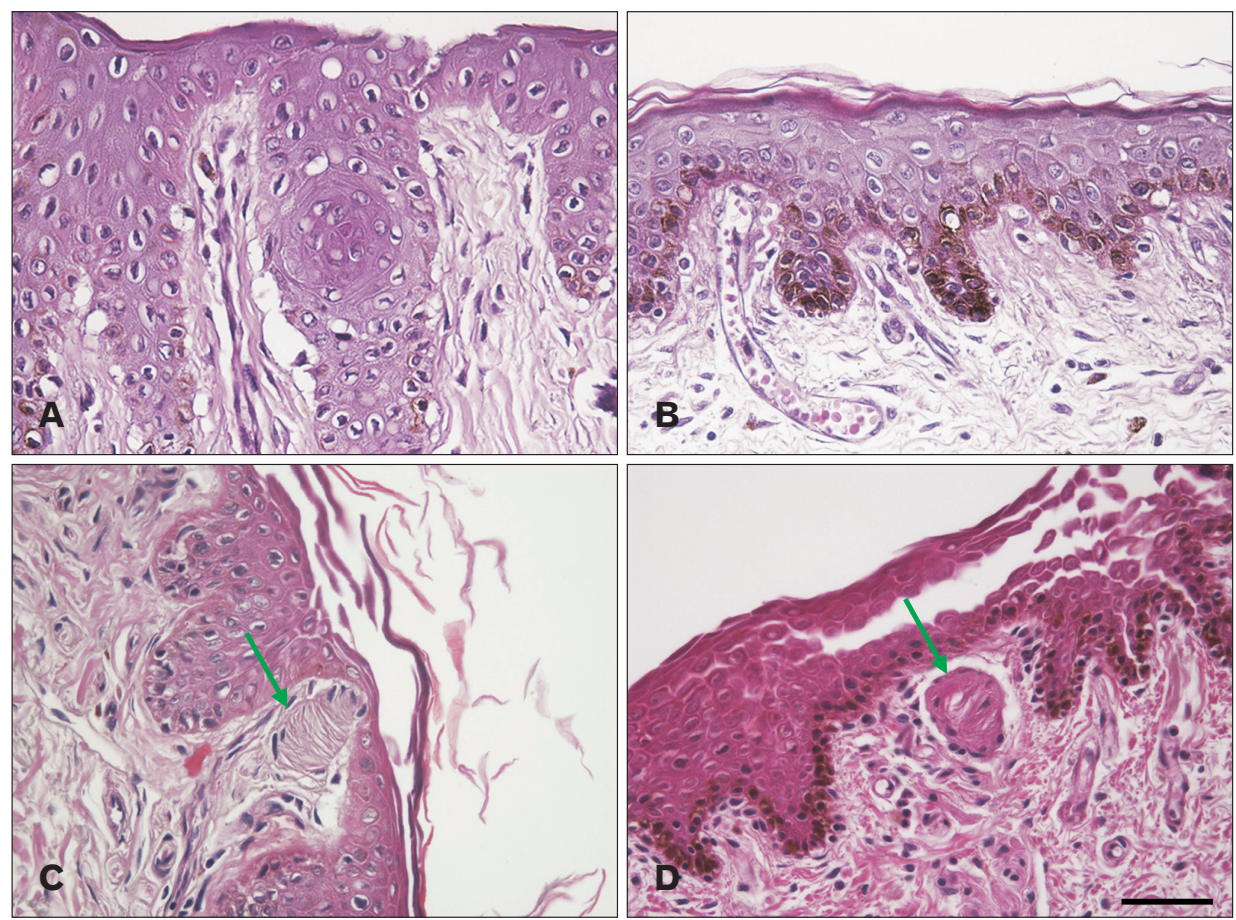

Fig. 2. Meissner's corpuscle was not observed in the dermal papillae of the nipples (A) and labia majora (B). There were few Meissner's corpuscle-like structures in the dermal papillae of the lips (C) and labia minora (D) (arrow) Meissner's corpuscle-like structure, Scale bars $=50 \mu \mathrm{m}$.

\section{Discussion}

We found many Meissner's corpuscles in fingertips and palms, but few in the skin of the lips, nipples, and female external genital organs. Most studies of the distribution of Meissner's corpuscles have focused on the human hand. Nolano et al. [12] reported $33 / \mathrm{mm}^{2}$ Meissner's corpuscles in the fingertips, whereas Kelly et al. [13] reported $2.7 / \mathrm{mm}^{2}$ in the fingertips and $1.33 / \mathrm{mm}^{2}$ in the palms. Our results were closer to those of Kelly et al. [13] than those of Nolano et al. [12].

We used skin samples taken from aged females (average: 67.3). The density of Meissner's corpuscles is decreased by aging. The number of Meissner's corpuscles in the fingertips was decreased approximately $0.05 / \mathrm{mm}^{2}$ per year in our previous study (in the article of Master degree of the corresponding author). Therefore, the number of Meissner's corpuscles in the hands, lips, nipples, and external genital organs in our study might be lower than that of young people. However, the density change in Meissner's corpuscles by aging may not influence crucially the density pattern in Meissner's corpuscles among the regions in human body.

Our results differed from the reported distribution of Meissner's corpuscles, which includes the dermal papillae of the hairless skin of the hands, feet, lips, eyelids, nipples, and genital organs $[1-3,6,14]$. There are unquestionably many Meissner's corpuscles in the fingertips and palms, but the distribution of Meissner's corpuscles in the lips, eyelids, nipples, and genital organs requires re-evaluation. We found very few Meissner's corpuscles other than in the fingertips and palms, suggesting that Meissner's corpuscles have little role in tactile sensation, including two-point discrimination in lips, nipples, and genital organs.

To understand the distribution and function of human Meissner's corpuscles, it is helpful to analyze their distribution in other animals. These corpuscles are found in the soles of most animals' feet; in the cat's paw, they are present in the foot pad [15]. Meissner's corpuscles have been found in the glabrous skin of primates, including the fingertips [16]. Interestingly, the tip of the elephant's trunk, which plays an elaborate role in grasping objects, contains many Meissner's corpuscles. Moreover, increased corpuscle density is correlated with the extent to which primates are frugivorous $[16$, 17].

Schwann cells are stacked parallel to the skin in a lamellar structure with nerve fibers located between them in Meissner's corpuscle. The Schwann cells and nerve fibers are encapsulated and the capsule is connected to the surrounding epidermal layer by collagen fibers $[5,18]$. Therefore, Hoffmann et al. [17] argued that Meissner's corpuscles detect friction and deformation of the outer surface, providing enhanced tactile perception. Kandel et al. [19] insisted that 
Merkel discs, and not Meissner's corpuscles, respond most sensitively to texture and that the main function of Meissner's corpuscle is to detect stroking and fluttering sensations.

Meissner's corpuscle functions primarily in two-point identification. As measured using a two-point discriminator, the fingertips are the most sensitive area followed by the palms, which have fewer Meissner corpuscles than the fingertips. Braille can only be read using the fingertips, especially the centers of the fingertips, which have the highest concentrations of Meissner's corpuscles and Merkel discs [19]. The fingertips are sometimes referred to as the secondary eye.

We confirmed that there are more Meissner's corpuscles in the palms than in the lips. This suggests that the palms are capable of better two-point discrimination than the lips; however, the opposite is true [19]. Therefore, it may be too simple to say that Meissner's corpuscles are responsible for two-point discrimination.

Meissner's corpuscles are rapidly adapting mechanoreceptors, whereas the Merkel discs are slowly adapting mechanoreceptors $[1,19]$. Both the tip of an elephant's trunk and human fingertips, both of which contain many Meissner's corpuscles, are highly movable and therefore require dynamic tactile sensation, including two-point discrimination. As rapidly adapting mechanoreceptors, Meissner's corpuscles could play a role in dynamic tactile sensation. Zimmerman et al. [3] reported that Meissner's corpuscles were sensitive to movement across the skin. The human fingertips also have many Merkel discs $[3,19]$. The standard two-point discrimination test is a static, not a dynamic, test. Therefore, the sensitivity measured by this test may be determined mainly by the distribution of slowly adapting Merkel discs rather than Meissner's corpuscles.

The accepted explanation for the distribution and function of Meissner's corpuscles, i.e., that Meissner's corpuscles are distributed in the fingers, toes, palms, soles, lips, eyelids, nipples, and genital organs, may require modification. Unlike the mobile hands and feet, the lips, eyelids, nipples, and genital organs may not require rapidly adapting mechanoreceptors. Their two-point discrimination ability might be primarily due to other mechanoreceptors, such as Merkel discs. To prove this, it is necessary to examine the distribution of Merkel discs. In a future study, we will compare the distributions of Meissner's corpuscle and that of Merkel discs using immunohistochemical staining in various skin tissue $[20,21]$.

In conclusion, many Meissner's corpuscles are found in the fingertips and palms, but few in the lips, nipples, and external genital organs. Considering that Meissner's corpuscles are rapidly adapting mechanoreceptors, they may detect quick changes in tactile sensation, including two-point discrimination, in the movable (i.e., dynamic) glabrous skin. Therefore, Meissner's corpuscles in non-movable glabrous skin might be rare compared to their distribution in the fingertips and palms.

\section{ORCID}

Sang Hyun Kim: https://orcid.org/0000-0001-9692-4838

Young Ho Lee: https://orcid.org/0000-0001-8238-2345

\section{Author Contributions}

Conceptualization: YHL. Data acquisition: SHK. Data analysis or interpretation: SHK, YHL. Drafting of the manuscript: SHK, YHL. Critical revision of the manuscript: YHL. Approval of the final version of the manuscript: all authors.

\section{Conflicts of Interest}

No potential conflict of interest relevant to this article was reported.

\section{Acknowledgements}

This work was supported by research fund of Chungnam National University.

We thank Min Geun Chee, Sa Deok Hong, and Soo Min Kim for observing Meissner's corpuscles in many tissue slides for our study.

\section{References}

1. Snell RS. Clinical neuroanatomy. 7th ed. Philadelphia: Lippincott Williams \& Wilkins; 2010.

2. Singh I. Textbook of human histology (with colour atlas \& practical guide). 6th ed. New Delhi: Jaypee Brothers Medical Publisher; 2011

3. Zimmerman A, Bai L, Ginty DD. The gentle touch receptors of mammalian skin. Science 2014;346:950-4.

4. Glimans S. Tactile corpuscle [Internet]. San Francisco: Wikipedia; 2017 [cited 2020 Apr 25]. Available from: https:// en.wikipedia.org/wiki/Tactile_corpuscle.

5. Castano P, Rumio C, Morini M, Miani A Jr, Castano SM. 
Three-dimensional reconstruction of the Meissner corpuscle of man, after silver impregnation and immunofluorescence with PGP 9.5 antibodies using confocal scanning laser microscopy. J Anat 1995;186(Pt 2):261-70.

6. Martín-Alguacil N, Cooper RS, Aardsma N, Mayoglou L, Pfaff D, Schober J. Terminal innervation of the male genitalia, cutaneous sensory receptors of the male foreskin. Clin Anat 2015;28:385-91.

7. Vega JA, López-Muñiz A, Calavia MG, García-Suárez O, Cobo J, Otero J, Arias-Carrión O, Pérez-Piñera P, Menéndez-González M. Clinical implication of Meissner's corpuscles. CNS Neurol Disord Drug Targets 2012;11:856-68.

8. Idé C, Saito T. Electron microscopic histochemistry of ATPase and alkaline phosphatase activities in mouse digital corpuscles. J Neurocytol 1980;9:207-18.

9. Matsuoka S, Suzuki H, Morioka S, Ogawa Y, Kojima T. Quantitative and qualitative studies of Meissner's corpuscles in human skin, with special reference to alterations caused by aging. J Dermatol 1983;10:205-16.

10. Crowley M, Lilak A, Ahloy-Dallaire J, Darian-Smith C. Spinal cord injury transiently alters Meissner's corpuscle density in the digit pads of macaque monkeys. J Comp Neurol 2019;527:1901-12.

11. Bolton CF, Winkelmann RK, Dyck PJ. A quantitative study of Meissner's corpuscles in man. Neurology 1966;16:1-9.

12. Nolano M, Provitera V, Crisci C, Stancanelli A, WendelschaferCrabb G, Kennedy WR, Santoro L. Quantification of myelinated endings and mechanoreceptors in human digital skin. Ann Neurol 2003;54:197-205.

13. Kelly EJ, Terenghi G, Hazari A, Wiberg M. Nerve fibre and sen- sory end organ density in the epidermis and papillary dermis of the human hand. Br J Plast Surg 2005;58:774-9.

14. Taylor JR, Lockwood AP, Taylor AJ. The prepuce: specialized mucosa of the penis and its loss to circumcision. Br J Urol 1996;77:291-5.

15. Bolanowski SJ, Pawson L. Organization of Meissner corpuscles in the glabrous skin of monkey and cat. Somatosens Mot Res 2003;20:223-31.

16. Verendeev A, Thomas C, McFarlin SC, Hopkins WD, Phillips KA, Sherwood CC. Comparative analysis of Meissner's corpuscles in the fingertips of primates. J Anat 2015;227:72-80.

17. Hoffmann JN, Montag AG, Dominy NJ. Meissner corpuscles and somatosensory acuity: the prehensile appendages of primates and elephants. Anat Rec A Discov Mol Cell Evol Biol 2004;281:1138-47.

18. Feito J, García-Suárez O, García-Piqueras J, García-Mesa Y, Pérez-Sánchez A, Suazo I, Cabo R, Suárez-Quintanilla J, Cobo J, Vega JA. The development of human digital Meissner's and Pacinian corpuscles. Ann Anat 2018;219:8-24.

19. Kandel ER, Schwartz JH, Jessell TM. Principles of neural science. 4th ed. New York: McGraw-Hill; 2000.

20. Albuerne M, De Lavallina J, Esteban I, Naves FJ, Silos-Santiago I, Vega JA. Development of Meissner-like and Pacinian sensory corpuscles in the mouse demonstrated with specific markers for corpuscular constituents. Anat Rec 2000;258:235-42.

21. García-Mesa Y, García-Piqueras J, García B, Feito J, Cabo R, Cobo J, Vega JA, García-Suárez O. Merkel cells and Meissner's corpuscles in human digital skin display Piezo2 immunoreactivity. J Anat 2017;231:978-89. 